\title{
PARTICIPACIÓN DE LAS MUJERES EN LAS UNIDADES PRODUCTIVAS FAMILIARES RURALES, ROSITA, RAAN
}

Sonia del Carmen García Aguilar ${ }^{[1]}$ Argentina García Solórzano ${ }^{[2]}$

\section{Resumen}

Se identificó el nivel de desempeño de las mujeres en las Unidades Productivas Familiares (UPF) y la relación existente entre las familias de la comunidad el "Zopilote", municipio de Rosita, Región Autónoma Atlántico Norte.

El estudio es cualitativo, se han descrito situaciones, personas, interacciones, actitudes y comportamientos observables. Los fundamentos teórico-metodológicos tienen como enfoque el interaccionismo simbólico porque se reflexiona sobre los significados sociales que las personas asignan al mundo que les rodea para valorar o visibilizar la participación de las mujeres en el trabajo y en la toma de decisiones en las UPF. Las técnicas fueron entrevistas, observación participante, grupos focales, mapeo de las unidades productivas seleccionadas y revisión documental.

Se encontró que las mujeres de la comunidad el "Zopilote" están involucradas en diferentes actividades dentro de las Unidades Productivas Familiares. Tienen un alto nivel de participación en todo el ciclo productivo, evidenciándose así que tienen parte en algunas decisiones y que estas son menores sobre los rubros de mayor valor para la comercialización de los productos. El estudio aporta la facilitación de nuevas estrategias a todas las ONG e instituciones del Estado que desean trabajar en la comunidad con temas relacionados al estudiado.

Palabras clave: Mujeres, unidades productivas, interaccionismo, estrategias.

\section{Summary}

We identified the level of performance of women in the Family Production Units (FPU) and the relationship between the families of the community the "Zopilote", municipality of Rosita, North Atlantic Autonomous Region.

\footnotetext{
[1] Máster en Desarrollo con Identidad con mención en Territorialidad, gobernabilidad y manejo de bosque: sonya311274@yahoo.es

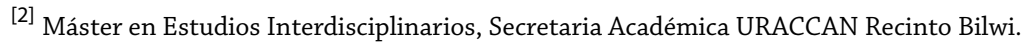
argentina_garcia@yahoo.com
} 
The study is qualitative and it describes situations, people, interactions, attitudes and observable behaviors. The theoretical and methodological approach are focus on a symbolic interactionism because it examines the social meanings that people put on the world that surrounds them to assess and highlight women participation in the work and decision-making in the FPU. The techniques that were applied are: interviews, participant observation, focus groups, mapping of the selected production units and document review.

Among the results we found that the women of the community the "Zopilote" are involved in different activities within the family production units. They have a high level of participation in the entire production cycle, and this is evident that they are taken into consideration in some decisions which are less on the items of greater value for the marketing of products. The study provides new strategies to the NGO's and state institutions who are interested to work in the community on issues related to this study.

Keywords: Women, productive units, interactionism, strategies.

\section{Introducción}

Esta investigación estudia el desempeño de las mujeres en las áreas productivas de trabajo, el entorno socioeconómico y ambiental del área de estudio y la relación que existe entre las familias. Se desarrolló entre septiembre y noviembre del 2010 en la comunidad el "Zopilote", que responde administrativamente al municipio de Rosita, Región Autónoma del Atlántico Norte, comunidad mestiza.

Se analizó la importancia que tiene la participación de las mujeres, no obviando el apoyo que les brindan sus hijos e hijas en las unidades productivas familiares, como generadoras de ingresos, beneficios satisfactorios para ellas y sus familias. Conscientes que el trabajo desempeñado por las mujeres en las unidades productivas ha tenido una importancia vital desde la prehistoria, aunque su contribución a la economía ha variado según la estructura, las necesidades, las costumbres y los valores sociales en la actualidad.

Se identificó una alta participación de mujeres y niños en las actividades productivas y domésticas de las Unidades Productivas Familiares, así mismo se identificó un bajo nivel en la toma de decisiones de las mujeres en la comercialización de algunos productos obtenidos del trabajo que realizan en las UPF. La participación de niñas y niños es de gran ayuda para poder desarrollar el trabajo de las mujeres en las parcelas y en el hogar. 
Se aportan resultados significativos para el desarrollo de nuevos proyectos, sobre las mujeres y la niñez, considerando la educación de los menores como el objetivo principal de las familias en la comunidad.

\section{Revisión de literatura}

Territorio: Arteaga \& Solís (2005:221), establecen que territorio es un espacio delimitado que incluye una relación de poder por parte de un individuo o grupo social. Comunidad: Turner, V. (1969) plantea: "Por lo general en una comunidad se crea una identidad común, mediante la diferenciación de otros grupos o comunidades (generalmente por signos o acciones) que es compartida y elaborada entre sus integrantes y socializada".

Participación, Según Oakley (1991:179): "La participación ciudadana desafía cualquier intento de definición o interpretación." Y según Cunill (1991) ha sido definida como "la intervención de la sociedad civil en la esfera de los público".

Mujer: La FAO (2004:1) plantea: "la contribución de las mujeres en la agricultura está ampliamente subestimada, las consideran trabajadoras familiares no remuneradas a pesar de su participación en todo el ciclo productivo".

Marco legal de protección de la niñez y adolescentes trabajadores: La normativa relacionada con el trabajo infantil en Nicaragua está contemplada en la Constitución Política, Código de Trabajo, Código de la Niñez y la Adolescencia, Código Penal.

Finca: Fundo o predio, es una propiedad inmueble que se compone de una porción delimitada de terreno (Enciclopedia Libre; 2005).

Unidades Productivas: Según Valdez Luis R. (2007:177), son un tipo particular de determinadas áreas de terrenos, manejadas por familias campesinas donde se produce bienes y servicios, en los cuales sus productos satisfacen necesidades humanas fundamentales de la vida diaria.

\section{Materiales y método}

La estrategia metodológica es el estudio de caso. Las técnicas definidas son las entrevistas abiertas, diálogo con la niñez de forma individual y grupal, entrevistas a mujeres, grupos focales, y la observación participante. Los instrumentos fueron las guías de las técnicas señaladas, grabadoras, GPS, papelógrafos y libreta de campo.

Para su desarrollo se tomaron en cuenta algunas variables: Actividades de la mujer en las Unidades Productivas Familiares, nivel de participación de la mujer en 
la toma de decisión, participación de niñas y niños. Para el desarrollo del trabajo se consideraron indicadores sociales, culturales y económicos.

De 410 habitantes se seleccionaron a 18 mujeres de las UPF y 36 niños y niñas.

\section{Aspecto ético}

La información y datos obtenidos no serán utilizados para fines lucrativos sino que tienen el propósito de que los resultados contribuyan para el desarrollo de mejores oportunidades y espacios de desempeño para las mujeres en el trabajo productivo.

\section{Resultados y discusión}

El presente estudio sobre las mujeres y unidades productivas refleja los siguientes resultados:

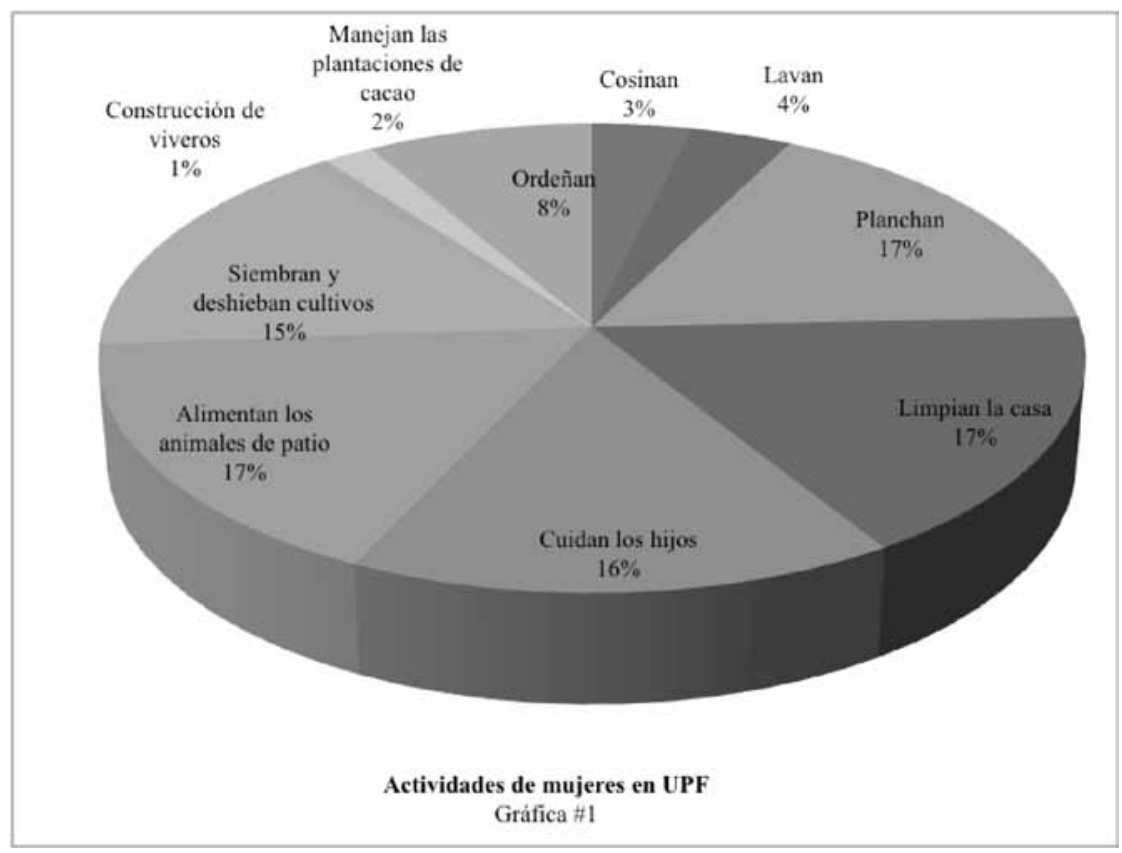

Gráfico No. 1. Actividades que las mujeres realizan en la Unidades Productivas Familiares.

Las mujeres de la comunidad el "Zopilote" están involucradas en diferentes actividades en las Unidades Productivas Familiares. Es oportuno mencionar que las mujeres trabajan todos los días tanto en el hogar como en las parcelas destinadas a la producción de sus alimentos que a continuación se aprecian. 
En la gráfica $\mathrm{N}^{\circ}$ 1. se reflejan las actividades principales que realizan las mujeres en las Unidades Productivas Familiares (UPF) de la comunidad el "Zopilote". El 17 \% limpia la casa, plancha y alimenta a los animales de patio, un $16 \%$ cuida a sus hijos, el 15\% trabaja en el campo en siembra y deshierbas de los cultivos en las UPF, el $8 \%$ labora en ordeño, el $4 \%$ lava ropa de toda la familia, el $3 \%$ preparan la alimentación, $2 \%$ se encarga de manejar las plantaciones de cacao en sus UPF, y $1 \%$ trabaja en construcción de viveros.

La señora López Dávila expresa:

Soy la cabeza de la familia, realizo todo tipo de siembra en mi finca con ayuda de mis hijos, siembro granos básicos, tubérculos, musáceas, cacao, manejo cerdos, gallinas, pelibuey, unas cuantas vaquitas, caballo, de lo que produzco de la finca es con lo que hasta la fecha mantengo a mis hijos, los quehaceres del hogar los realizo, junto a mi nuera, para luego ir a trabajar a las parcelas donde tenemos las siembras.

La FAO (2004), destaca la elevada contribución de las mujeres en las labores agrícolas pero que está ampliamente subestimada, y son consideradas trabajadoras familiares no remuneradas a pesar de su participación en todo el ciclo productivo. El BID (1991), señala que ha existido la tendencia de subestimar la función económica de la mujer y su participación, reconoce que se debe destacar más su contribución actual y potencial como productora, como individuo capaz de tomar decisiones y como generadora de ingresos.

La gráfica No. 2, muestra productiva de las mujeres en las UPF; el 39 \% leche y maíz son los principales productos a los cuales se les aplica una transformación, seguido del coco, y $2 \%$ cacao y barro.

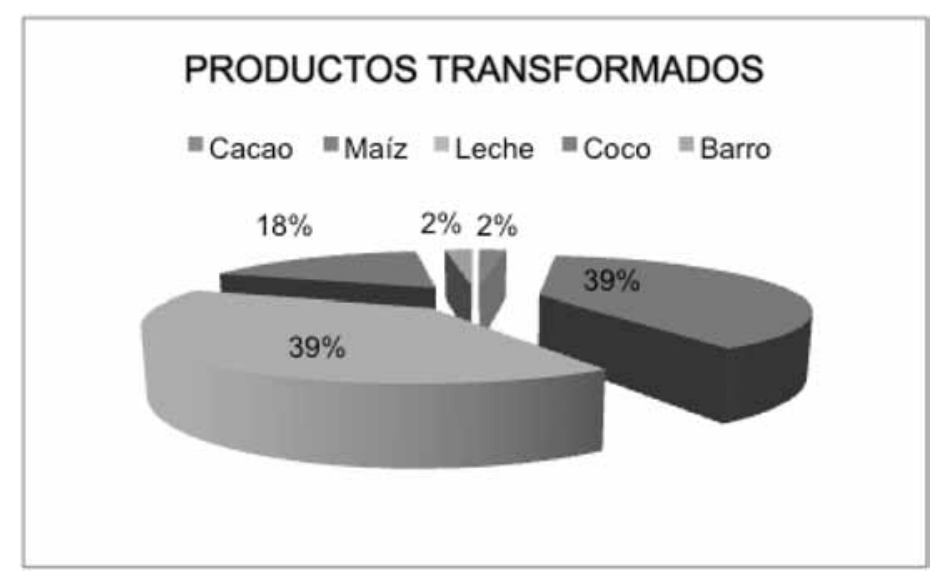

Gráfico No. 2. Porcentaje de producción de las mujeres. 
Una vez transformado el maíz, obtienen productos como: horneados de maíz, pinolillo, pozol, atol, tamales, tortillas; de la leche sacan cuajadas, crema, queso, cajetas de leche, del coco producen las cajetas de coco y del barro hacen loceras. De todos estos productos que transforman, solamente a uno de ellos se le da valor agregado como es el cacao, convertido en chocolate, el cual es ofrecido al consumidor con un empaque, generando un ingreso mayor para la economía familiar.

Confirma esto la Señora González Ruiz cuando dijo: "Yo soy la persona que se encarga de hacer los chocolates y hago todo el proceso para poderlo llevar a los consumidores, soy la única en esta comunidad que hace este tipo de trabajo".

Según algunas mujeres entrevistadas los productos que se comercializan en las UPF son: maíz, frijol, cuajadas, gallina, cerdos, banano, yuca, arroz, huevos, pejibaye, naranja, mango, coco, aguacate, y chocolates. Otros productos que contribuyen a la economía de las UPF son los frijoles y el maíz, resolviendo en la temporada de cosechas. Otros productos de menor comercialización: cuajadas, aguacate, huevos, gallina, cerdos, bananos, yuca, mango, coco y chocolates. El $80 \%$ de los productos son vendidos en la comunidad a intermediarios, el $20 \%$ son llevados a Rosita, directamente al consumidor.

Cuadro No. 1. Horas trabajadas (HT) por las Mujeres en las unidades productivas familiares

\begin{tabular}{|l|c|l|c|}
\hline \multicolumn{1}{|c|}{ Hogar } & H T & \multicolumn{1}{|c|}{ Parcelas } & H T \\
\hline Cocinar & 3 & $\begin{array}{l}\text { Siembra granos y tubér- } \\
\text { culos }\end{array}$ & 3 \\
\hline Limpiar & 1 & Siembra de Hortalizas & 2 \\
\hline Lavar & 2 & Construcción de viveros & 2 \\
\hline Dar comida a animales & 1 & Manejo del cacao & 2 \\
\hline Jala agua & 1 & Deshierbe de Maíz, frijoles & 2 \\
\hline Cuidar los hijos & 8 & & $\mathbf{1 1}$ \\
\hline Total horas & $\mathbf{1 6}$ & Total horas & \\
\hline
\end{tabular}

El cuadro No.1 nos muestra la cantidad de horas trabajadas por las mujeres en el hogar. Se encontró que 16 horas diarias son dedicadas al trabajo doméstico, inician sus labores desde las 4 de la mañana y finalizan a las 8 de la noche. 11 horas son dedicadas a las labores de producción en las parcelas, es lo que realizan en temporada de siembra y cosecha de algunos granos básicos; cuando no es tiempo de cosecha y siembra, laboran solamente 4 horas por semana, es decir que siempre la mujer tiene una doble jornada laboral, la cual no es reconocida.

Se puede observar que el tiempo y el trabajo destinado a labores domésticas de las mujeres, muestran una tendencia en términos generales, a un mayor aporte de horas diarias de trabajo. Puede decirse que las mujeres llevan a cabo una función muy 
importante como reproductoras de la unidad familiar campesina, desempeñan labores que como esposas, madres, amas de casa y sin las cuales sería imposible reproducir la fuerza de trabajo en el medio rural solo por el hombre. Además, se vinculan como trabajadoras de la finca o la parcela durante todo el año, en la mayoría del ciclo productivo así como agregando valor a la materia prima.

En un estudio de la FAO (2004) refleja que las mujeres participan en la agricultura tanto o más que los hombres en la siembra y más ampliamente en el levantamiento de la cosecha y post-cosecha. Como podemos notar, es amplia la participación, pero eso no es reconocido social y monetariamente es menos menor con relación a la paga que reciben los hombres. Es claro el aporte de estas mujeres a la economía familiar.

\section{Nivel de participación de la mujer en la toma de decisiones de las UPF}

Las mujeres en la Comunidad el "Zopilote" a diario trabajan en sus Unidades Productivas Familiares. En la gráfica No. 2 se aprecian los productos para la comercialización que las mujeres tienen acceso a tomar decisiones en las UPF.

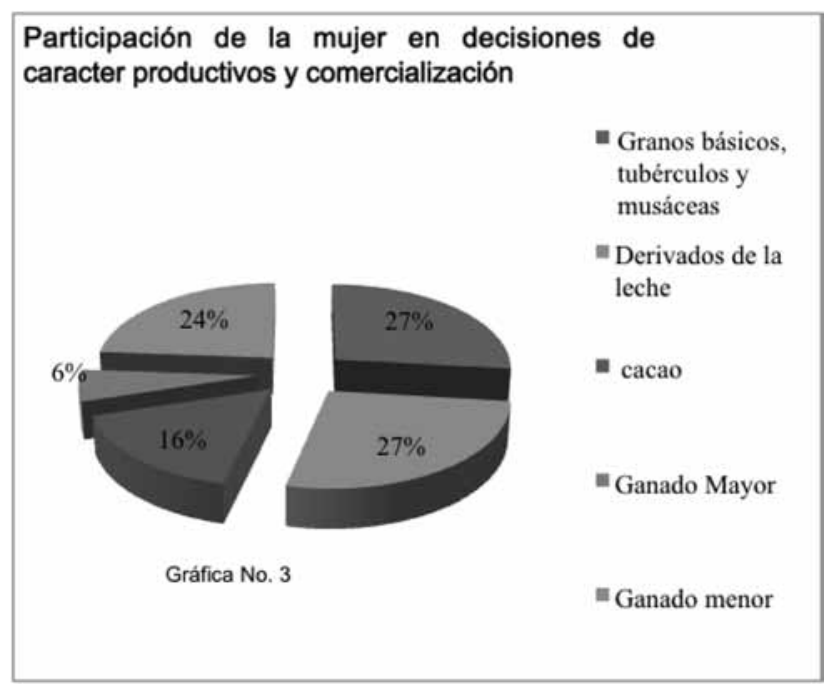

Gráfico No. 3. Porcentaje en la toma de decisiones de las mujeres.

En la gráfica No.3. se observa que el nivel de participación es alto en todo el ciclo productivo, evidenciándose de esta manera, la participación que tiene la mujer en decisiones a la hora de comercializar productos de las UPF. El $27 \%$ de las mujeres participan en la toma de decisión a la hora de la comercialización de la leche y sus derivados; granos básicos, tubérculos y musáceas, el $24 \%$ en ganado menor, el $16 \%$ cacao, el $6 \%$ ganado mayor. 
Señala Merino (1995) que la participación significa lo que hacen las personas para afectar la toma de decisiones, ejecución y seguimiento de las decisiones públicas. La (OIT) define Trabajo Decente como la libertad para que los individuos manifiesten sus preocupaciones, se organicen y participen en la toma de aquellas decisiones que afectan a sus vidas, así como la igualdad de oportunidades. Los resultados encontrados dicen lo contrario ya que las mujeres de las Unidades Productivas Familiares, no tienen esa libertad de decidir sobre algunos rubros a comercializar, aunque tienen participación activa en todo el trabajo que se desarrolla en las UPF.

Como trabajadoras, a la hora de la comercialización no tienen la oportunidad de decidir sobre los rubros que tienen mayor valor, siempre les dejan comercializar los productos de menor valor; corroborando de esta manera que las mujeres participan, pero no todas tienen la oportunidad en la toma de decisión.

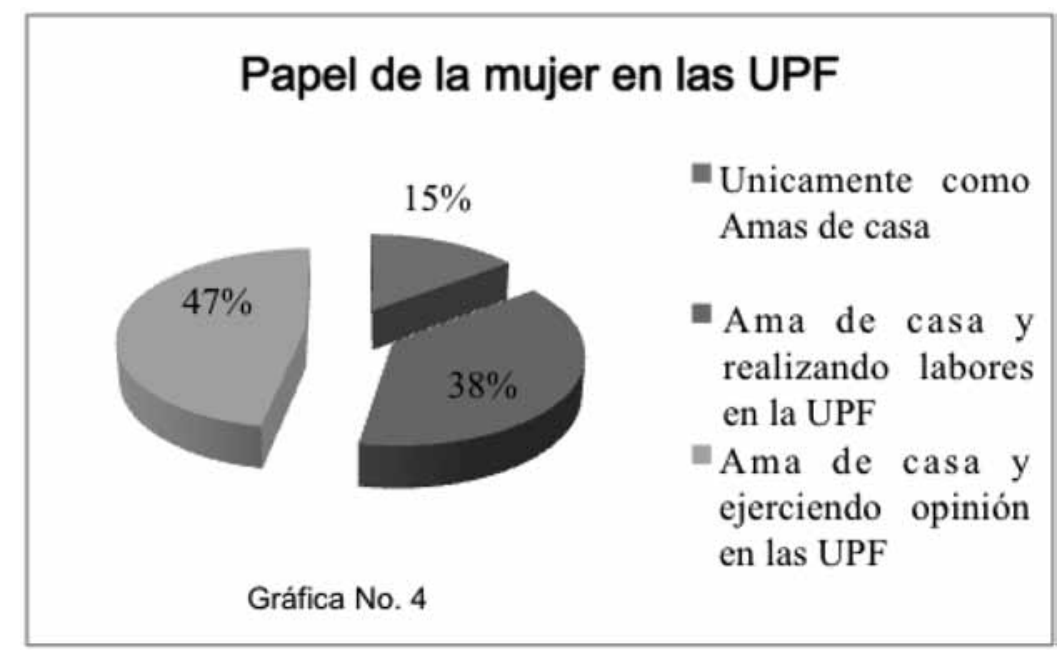

Gráfico No. 4. Porcentaje del papel de las mujeres en la UPF.

En la gráfica No. 4. se refleja el papel de la mujer en las UPF. El $47 \%$ trabaja como amas de casa y ejercen opinión en las UPF, el $38 \%$ como amas de casa y realiza labores en las UPF, y el 15\% únicamente como amas de casa.

El Centro para la Investigación Forestal Internacional afirma que los roles de las mujeres en las familias es estratégico para la subsistencia, contribuyen con su trabajo de manera significativa a la producción de alimentos y de algunos excedentes, también asumen la totalidad de las labores reproductivas: lactancia, preparación de alimentos, acarreo de agua, recolección de leña, limpieza y cuidado de los niños, atención a los enfermos y otros.

La señora Martínez Urbina expresó: "A mí simplemente no me gusta trabajar en el campo por eso solo hago los quehaceres del hogar". Contrario a este comentario la 
señora Acosta Ochoa dice: "yo ayudo a mi marido en las siembras de yuca, frijoles y a deshierbar ya que mis niños están pequeños y no nos pueden ayudar". Pero doña Vicenta Martínez García comenta: "A mí siempre me ha gustado vivir en el campo, desde pequeña me gusta trabajar, sembrar y nadie me obliga hacerlo, sino que es decisión mía porque me gusta".

Ante estas opiniones y resultados de la gráfica No. 4, puede deducirse que no todas las mujeres de la comunidad el "Zopilote" tienen la oportunidad de decidir qué hacer y qué no hacer, ante todo las mujeres son amas de casa y les guste o no les guste tienen que hacer el trabajo que realizan en sus UPF para sobrevivir, aunque algunas juegan un papel muy importante como las que participan y toman decisiones.

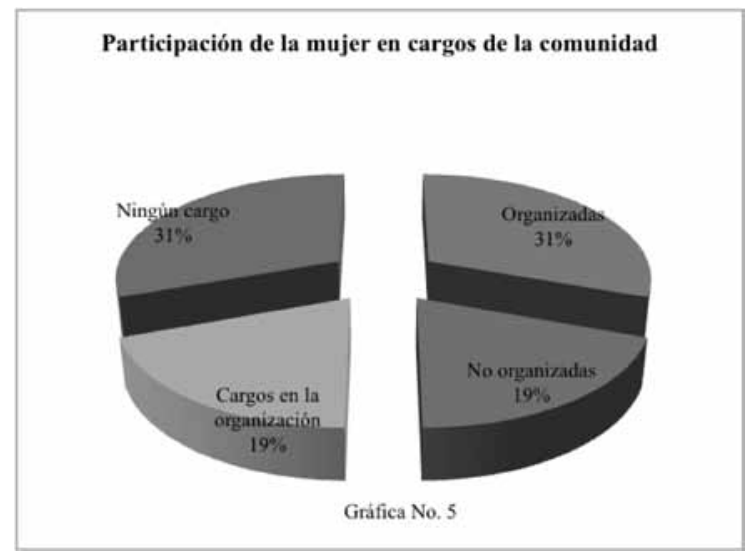

Gráfico No. 5. Porcentaje de la participación de las mujeres en la comunidad.

En la gráfica No. 5 se ve reflejada la participación que tienen las mujeres en diferentes cargos en la comunidad. Un 31 \% están organizadas dentro de la comunidad y con las diferentes ONG que les apoyan, un $19 \%$ no están organizadas con nadie, el $31 \%$ no tiene ningún cargo en la comunidad y el 19 \% goza de cargos dentro de la comunidad y a nivel de ONG, como mujeres organizadas.

Arteaga \& Solís (2005), establecen que territorio es un espacio delimitado que incluye una relación de poder por parte de un individuo o grupo social. Implica referirse a límites de soberanía, propiedad, apropiación, control y jurisdicción. El territorio trasmite la idea de delimitación y dominio de un espacio. También está relacionado con prácticas ligadas con el poder público, estatal o privado en todas sus escalas, por lo que tiene la función de gestión de un espacio determinado.

Dos definiciones parecidas donde el término territorio no está separado de comunidad, más bien comunidad es parte del territorio, en donde se ponen de manifiesto muchas características como dominio de su espacio en donde se vive, la participación 
que tienen los individuos en diferentes prácticas relacionadas con el poder público, como los resultados lo indican en la gráfica $\mathrm{N}^{\circ} \cdot 5$.

Puede decirse que las mujeres de la comunidad el "Zopilote" tienen una representación mínima en cuanto a cargos se refiere, no hay una representación en las estructuras que tiene la comunidad, la presencia de las mujeres es escasa, ante cualquier cargo público que tenga la comunidad, claramente se ve que las mujeres están organizadas gracias a las ONG que trabajan con ellas.

\section{Incidencia en menores de 12 años en actividades de las UPF}

Las niñas y niños realizan diversas actividades en las UPF, lo que de una u otra forma son actividades que las realizan en su mayoría contra su voluntad. Jadder Flores López de 9 años de edad, cursa el primer grado, en su hogar solo tiene la figura materna al preguntarle que hacía en su hogar y si le gustaba lo que hacía contesta: "No me gusta lo que hago, no me gusta trabajar, yo quiero jugar y estudiar".

Parecidas otras expresiones de algunas niñas y niños al hacerles la misma pregunta, como es el caso de las niñas: Maribel Pérez Mendoza y Mary Luz Aguilar Suárez, la primera de 8 años y las segunda de 9 años, ambas viven con su papá y mamá, y comentan: "A nosotros nos mandan a realizar algunos trabajos y si no lo hacemos nos castigan. Pero nos gustaría jugar y estudiar".

El resto de la niñez opinó de una forma diferente, los mayores de 10 a 12 años, consideran que es responsabilidad de ellos ayudar a sus padres en los quehaceres de las UPF.

Cuadro No. 2. Actividades que realizan los niños y niñas en las UPF

\begin{tabular}{|c|c|}
\hline Niños & Niñas \\
\hline Rajan leña & Barren \\
\hline Jalan leche & Lavan maíz \\
\hline Echan las vacas al corral & Venden Boli \\
\hline Jalan agua & Muelen maíz \\
\hline Entran leña & Lavan traste \\
\hline Limpian patio & Jalan agua \\
\hline Ordeñan & Cocinan \\
\hline Montan a caballo & Entran leña \\
\hline Chapean & Lavan ropa \\
\hline Hacen mandados & Limpian patio \\
\hline Ayudan en las siembras y deshierba & Ordeñan y encierran terneros \\
\hline
\end{tabular}




\begin{tabular}{|l|l|}
\hline \multicolumn{1}{|c|}{ Niños } & \multicolumn{1}{|c|}{ Niñas } \\
\hline Encierran terneros & Montan a caballo \\
\hline & Hacen cuajadas \\
\hline & Echan tortillas \\
\hline & Hacen mandados \\
\hline & Prenden fuego \\
\hline & Limpian inodoro \\
\hline & Ayudan en las siembras y deshierba \\
\hline
\end{tabular}

Niños y niñas que asisten a la escuela de la comunidad el Zopilote

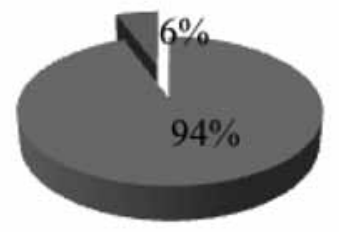

Asisten

No asisten

Gráfico No. 6

Gráfico No. 6. Porcentaje de asistencia de la niñez a la escuela el "Zopilote".

En la gráfica No.6 se reflejan las actividades que la niñez realiza en las UPF, mostrándose claramente que las actividades realizadas por los niños son unas y las actividades realizadas por las niñas son otras, son pocas las actividades en las que se involucran niños y niñas.

El $41.66 \%$ voluntariamente se involucra en las actividades de trabajo en las UPF, son niños y niñas que andan entre los 10 y 12 años. El $58.33 \%$, comentó que no todo el tiempo quieren ayudar en las UPF, y si lo hacen es por exigencias de sus padres, son menores que andan entre 6 y 9 años de edad. Se comprueba que la niñez de la comunidad el "Zopilote" están expuestos a estos tipos de trabajo, reconociendo así que los padres de familia creen que esa es la manera de prepararlos para su futuro.

Existe una normativa relacionada con el trabajo infantil en Nicaragua y está contemplada en la Constitución Política, el Código de Trabajo, el Código de la Niñez y la Adolescencia, el Código Penal, los Convenios de la OIT números 138 y 182, y el Acuerdo Ministerial núm. VGC-AM-0020-10-06 que contemplan como delitos la explotación laboral, el trabajo forzoso y el trabajo peligroso; diversos decretos para proteger a la 
niñez y adolescencia sobre el listado de trabajos peligrosos aplicado para el caso de Nicaragua, relativos a la edad mínima de admisión en el empleo y a la prohibición de las peores formas de trabajo infantil.

Como se puede apreciar, existen varias leyes que contemplan los derechos y deberes de la niñez, pero en la realidad en las zonas rurales no se aplicable. Si bien es cierto en la zona urbana existe una institución de gobierno llamada Ministerio de la Familia, su papel fundamental es velar por la unión de las familias, así mismo velar por los derechos y deberes de estos, conociéndose que el personal de esta institución es reducido y que principalmente atiende el casco urbano.

Las comunidades no tienen ninguna atención y pasan desapercibidas, y no se aprecia si los niños del campo son explotados, porque lo toman como natural que la niñez trabaje muchas horas, y tampoco el trabajo que realizan no es visto acorde a su edad. En la figura $\mathrm{N}^{\circ} .6$ se refleja que el $94 \%$ de los niños asisten a clase, el $6 \%$ no asiste a clase, claramente se manifiesta que es prioridad la educación para los niños y niñas en la comunidad el "Zopilote". Así lo expresó la señora González Rodríguez: "mis niños tienen que ir a clase, es mi responsabilidad que ellos se eduquen porque si yo no tuve esa oportunidad, por lo menos voy a luchar para que ellos no sean igual a mí".

La niñez de la comunidad el "Zopilote", en el caso de los más mayores sienten la necesidad y deber de ayudar en las UPF, es una cultura dentro de las familias campesinas involucrarse todos en los trabajos, pero a ojos de los dirigentes de la comunidad han habido cambios, de tal forma que los niños y niñas para que desempeñen una labor, ésta tiene que ir acorde a su edad y contextura, considerando que los niños tienen que ir aprendiendo las labores de las UPF de acuerdo a su edad.

\section{Estrategias que permitan un mayor nivel de toma de decisión de la mujer y reducción de las jornadas de trabajo en las unidades productivas familiares}

Las estrategias van orientadas a motivar cambios en la cultura, de tal forma que sean asumidas en la cotidianeidad, en las reglas socialmente compartidas.

Algunas estrategias pueden ser:

- Propiciar un acercamiento entre esposa - esposo e hijos, donde puedan conversar abiertamente sobre el trabajo que realizan todos en las UPF, considerando puntos de vista como el nivel de decisiones que debe tener la mujer, así mismo llegar a acuerdos para una mejor comprensión en cuanto al trabajo que realiza la mujer.

- Articular con las diferentes ONG e instituciones del Estado, que dan acompañamiento a las mujeres, tener un mayor acercamiento e involucrar a los hombres en las capacitaciones de género, de tal manera que los dos asistan a las capacitaciones que sean impartidas. 


\section{GÉNERO E IDENTIDAD}

- Plantear a la comunidad en asamblea se aborden temas, donde las mujeres puedan insertarse y participar en trabajos de la comunidad y así iniciar un proceso de cambio de actitud en los hombres.

- Plantear la necesidad de que la mujer asuma responsabilidades compartidas con su compañero de vida, y concientizar a los comunitarios de la importancia de la educación, para que se le facilite la entrada de ingresos y obtener mayores beneficios, contribuyendo a la economía familiar.

- Aprovechar las reuniones de padres de familia en el centro escolar de la comunidad el "Zopilote", para que las ONG y instituciones del Estado que de una u otra forma están vinculada con las niñas y niños, puedan tener un espacio de dialogo, con los padres, para que se tomen en cuenta las opiniones de las niñas y niños en los trabajos que ellos desarrollan en las UPF, de acuerdo a su edad.

- Todos los que trabajan con las niñas y niños unir esfuerzo y trabajar con un solo objetivo, para no duplicar esfuerzo, y así enseñarle a los niños sobre sus derechos y deberes que tienen en las UPF.

\section{Conclusiones y recomendaciones}

\section{Conclusiones}

Es oportuno mencionar que las características patriarcales de estas sociedades determinan que las mujeres asuman múltiples roles ocasionando que las opciones y el tiempo del que disponen para participar en las reuniones o en otras actividades sean poco significativas.

Las actividades que realizan no son reconocidas por nadie, la presencia de la mujer en las estructuras de organizaciones de la comunidad es nula, tienen escasa participación en la toma de decisiones. Si bien es cierto, la mujer en la Comunidad el "Zopilote", está presente en algunas organizaciones, pero su participación no es significativa. La baja integración de la mujer en las estructuras de la comunidad se debe en parte a factores de índole cultural y a su poca disponibilidad de tiempo.

Las mujeres de la comunidad el "Zopilote" realizan diversas actividades dentro de las UPF, como trabajo principal se encuentra el doméstico seguido de los trabajos en las parcelas. No todo es negativo, las mujeres han dado un paso significativo y lo más importante es que quieren seguir luchando para ser tomadas en cuenta.

Además reconocen que todavía les falta más tiempo, para sentirse en igualdad con sus compañeros de vida, y que a pesar de tener varios años trabajando de la mano con algunas ONG, abordando diferentes temas de género, no es lo suficiente, ya que lo que se ha logrado es disminuir un poco el machismo de sus compañeros. 
La intervención de la niñez en las Unidades Productivas Familiares, es un aporte valioso para el desarrollo de actividades que realizan las mujeres en las UPF, considerando a los niños y niñas mano derecha de la mujer en todo el desarrollo de actividades que realizan a diario en cada Unidad Productiva Familiar. La ayuda de la niñez en las labores de las UPF es tomada por los padres de familia como una preparación para su futuro, es la forma de ayudar a sus hijas e hijos a prepararse para su vida adulta.

\section{A mujeres que trabajan en las Unidades Productivas Familiares de la comunidad el "Zopilote"}

- Necesitan fortalecer el funcionamiento de las mujeres organizadas e integrar más mujeres, para asumir una posición de control, tener opiniones y ser escuchadas dentro del hogar y la comunidad.

\section{A las ONG e instituciones del Estado que trabajan con las mujeres de la comunidad el "Zopilote"}

- Creación de mecanismos eficientes y eficaces de intervención entre los diferentes organismos que acompañan a las mujeres, para no duplicar esfuerzos y aprovechar mejor los recursos utilizados

\section{A estructuras de la comunidad el "Zopilote"}

- Es importante que apoyen y fomenten la participación de las mujeres en espacios de poder dentro de las UPF, y a nivel de comunidad para que sean reconocidas y respetadas como ciudadanas, y ser humanos en igualdad de condiciones y con algo que aportar al desarrollo socioeconómico, a la conservación de su lengua, cultura, identidad étnica y tradiciones.

- Podrían promover la integración de hombres a las actividades de la mujer de una forma sutil y estratégica, para lograr una igualdad y así reducir el trabajo en que realizan las mujeres en las UPF.

\section{A las ONG e instituciones del Estado}

- Coordinar actividades afines y crear mecanismos de monitoreo para dar respuesta a situaciones que se presentan a diario en las comunidades en relación con la niñez.

- Capacitar a mujeres y hombres de la comunidad el "Zopilote", en temáticas que ayuden a cambiar la actitud entre ellos.

- A la alcaldía de la municipalidad de Rosita que tome en cuenta a la comunidad el "Zopilote", en sus planes estratégicos, para desarrollar proyectos, cuyos beneficiarios sean las mujeres y la niñez.

\section{A las instituciones del Estado que velan por la niñez}

- Del presupuesto asignado, contar con una partida presupuestaria para incluir a la comunidad el "Zopilote", y así poder dar atención a este grupo que es completamente vulnerable. 


\section{GÉNERO E IDENTIDAD}

\section{Lista de referencias}

Arteaga, C. y Solís, S. (2005). Necesidades sociales y desarrollo humano. Un acercamiento metodológico. UNAM/ENTS. Plaza y Valdez. México. pp: 221- 230.

Banco Interamericano de Desarrollo, (1991). A muhler, no desenvovimento.

Cunill, N. (1991). Participación Ciudadana. Editorial del CLAD. Venezuela.

Merino, M. (1995). La participación ciudadana en la democracia. IFE. Cuadernos de Divulgación de la Cultura Democrática, No. 4. México.

Oakley, P. (1991). Projets whit People. The practice of participation in rural development. Internacional Labour Office. London. UK.

Servicio de Género y desarrollo en Nicaragua. FAO. (2004) La mujer en la Agricultura, medio ambiente y la producción rural. Nicaragua

Toledo, V. M. (1996). Los ejidos y las comunidades: lugar de inicio del desarrollo sustentable en México. Revista de la Universidad de Guadalajara 6:28-34.

Turner, V. (1969). Estudio sobre rituales. Octubre 04, 2010, de http:// es.wikipedia. org/wiki/Comunidad.

Convenios de la OIT No. 138 y 182Chávez, J. (2007, Noviembre 23). Para erradicar el trabajo infantil hay que acabar con la pobreza, pero entre tanto hay que avanzar. Octubre 21, 2010, http://www.reluita.org/laboral/ministra_trabajo_nica.htm.

El Programa Internacional para la Erradicación del Trabajo Infantil (IPEC; 2008) de la Organización Internacional de Trabajo. (http://www.rel-uita.org/laboral/ ministra_trabajo_nica.htm).(05/05/2010).Comunidad es.wikipedia.org/wiki/

Enciclopedia Libre (2005). Acceso septiembre 2010 en http://es.wikipedia.org/wiki/ Finca

INIM, (2003). La mujer en la agricultura, medio ambiente y la producción rural. Octubre 30, 2010 de http://www.rlc.fao.org/es/desarrollo/mujer/situacion/pdf/nicarag.pdf.

Mora, L. (1997). La economía popular alternativa: una visión sobre el terreno. Agosto 23, 2010 de http://www.nodo5o.org/espanica/realidad.htm.

URACCAN, (1999). Metodología para el monitoreo de los recursos naturales en la Región Minera y zona de amortiguamiento de la reserva de la Biosfera, Región Autónoma del Atlántico Norte, Nicaragua. Septiembre 10, 2010, de http://www.rimisp.org/ webpage.php?webid=454

Valdez Luis R. (2007) Comunidades: Asociatividad y producción en el territorio. Acceso septiembre 2010 de http://www.eumed.net/libros/2007a/268/52.htm. 